\title{
High frequency of resistance to the drugs isoniazid and rifampicin among tuberculosis cases in the City of Cabo de Santo Agostinho, an urban area in Northeastern Brazil
}

\author{
Alta frequiência de resistência às drogas isoniazida e rifampicina \\ em tuberculose na Cidade do Cabo de Santo Agostinho, \\ uma área urbana do nordeste do Brasil
}

\author{
Marcilio Baliza $^{1,7}$, Artur Henrique Bach ${ }^{1}$, Gabriel Lobo de Queiroz ${ }^{1}$, Inês Cardoso Melo², \\ Maria Madileuza Carneiro ${ }^{3}$, Maria de Fátima Pessoa Militão de Albuquerque ${ }^{1}$, Philip Suffys ${ }^{4}$, \\ Laura Rodrigues ${ }^{5}$, Ricardo Ximenes ${ }^{6}$ and Norma Lucena-Silva ${ }^{1,8}$
}

\begin{abstract}
The objective of the present study was to investigate the frequency and risk factors for developing multidrug-resistant tuberculosis in Cabo de Santo Agostinho, PE. This was a prospective study conducted from 2000 to 2003, in which suspected cases were investigated using bacilloscopy and culturing. Out of 232 confirmed cases of tuberculosis, culturing and antibiotic susceptibility tests were performed on 174 . Thirty-five of the 174 cultures showed resistance to all drugs. The frequencies of primary and acquired resistance to any drug were $14 \%$ and $50 \%$ respectively, while the frequencies of primary and acquired multidrug resistance were $8.3 \%$ and $40 \%$. Previous tuberculosis treatment and abandonment of treatment were risk factors for drug resistance. The high levels of primary and acquired resistance to the combination of isoniazid and rifampicin contributed towards the difficulties in controlling tuberculosis transmission in the city.
\end{abstract}

Key-words: Tuberculosis. Drug resistance. Multidrug resistance. Risk factors.

\section{RESUMO}

0 objetivo do presente estudo foi investigar a freqüência e fatores de risco para o desenvolvimento de tuberculose multidroga resistente, na Cidade do Cabo de Santo Agostinho, PE. Este é um estudo prospectivo realizado entre 2000-2003 onde casos suspeitos foram investigados por baciloscopia e cultura. De 232 casos de tuberculose confirmados, 174 tiveram cultura e antibiograma realizados. Trinta e cinco das 174 culturas mostraram resistência a qualquer uma das drogas. A frequiência de resistência primária e adquirida a qualquer droga foi 14\% e 50\% respectivamente enquanto a frequiência primária e adquirida para multidroga resistência foi 8,3\% e 40\%. Tratamento prévio para tuberculose ou abandono de tratamento consistiu em fatores de risco para resistência a drogas. Os altos níveis de resistência primária e adquirida a combinação isoniazida e rifampicina contribuem para as dificuldades no controle da transmissão da tuberculose no Cabo.

Palavras-chaves: Tuberculose. Resistência a drogas. Multidroga resistência. Fatores de risco.

The increasing frequency of drug-resistant tuberculosis (TB), which has been leading to failure to respond to either isoniazid (INH) or rifampicin (RMP) is of great concern in relation to successful implementation of large-scale regional TB control programs. In Brazil, the first-line drugs for TB treatment in use since 1982 are INH and RMP for six months in association with pyrazinamide (PZA) for the first two months. The drugs are supplied by the government, without cost to the patient, and the treatment is based on self-administration.

The World Health Organization (WHO) defines multidrugresistant tuberculosis (MDR-TB) as TB involving resistance to at least INH and RMP. However, the Brazilian Consensus

\footnotetext{
1. Departamento de Imunologia, Centro de Pesquisas Aggeu Magalhães, Fundação Oswaldo Cruz. Recife, PE. 2. Secretaria de Saúde do Cabo de Santo Agostinho, Posto de Saúde Herbert de Souza, Cabo de Santo Agostinho, PE. 3. Laboratório Central da Secretaria de Saúde do Estado de Pernambuco, Recife, PE. 4. Departamento de Micobacterioses, Instituto Oswaldo Cruz, Fundação Oswaldo Cruz, Rio de Janeiro, RJ, 5. Department of Epidemiology of the London School of Hygiene Tropical Medicine, London, United Kingdom, 6. Departamento de Medicina Tropical, Universidade Federal de Pernambuco, Recife, PE. 7. Centro de Ciências da Saúde, Universidade Federal do Recôncavo da Bahia, Santo Antônio de Jesus, BA. 8. Laboratório de Biologia Molecular, Serviço de Oncologia do Instituto Materno Infantil Prof. Fernando Figueira, Recife, PE.

Financial Support: This work was supported by FACEPE grant number: 23-CSD-08/00-01/01-29; CNPq Brazilian TB-Network, grant number 62.0055/01-4-PADCT III/MILÊNIO. Address to: Dra Norma Lucena Silva. Centro de Pesquisas Aggeu Magalhães/Fundação Oswaldo Cruz. Campus da UFPE, Av. Moraes Rego, S/N, 50670-420 Recife, PE. Tel: 5581 9962-3975; Fax: 5581 3453-2449.

e-mail: nlucena@cpqam.fiocruz.br

Recebido para publicação: em 5/07/2007

Aceito em: 28/01/2008
} 
for Tuberculosis that was agreed in 1997 classifies MDR-TB as tuberculosis caused by Mycobacterium that is resistant to more than two drugs including INH and RMP ${ }^{11}$. Primary resistance refers to the presence of resistant isolates of Mycobacterium in patients who, in response to direct questioning, deny having had any prior anti-TB treatment; it is thus a consequence of transmission of resistant isolates. Acquired resistance is defined as the presence of resistant isolates of Mycobacterium in patient who, in response to direct questioning, admit having been treated for tuberculosis; it is thus due to selection of the bacterial population by development of mutation(s) that confer(s) survival advantages under antibiotic selective pressure ${ }^{15}$. The recommended treatment for multidrug-resistant tuberculosis is more toxic, takes at least three times longer and is a hundred times more expensive than a short course of treatment. It is also less successfull ${ }^{16}$.

In 2004, WHO published a survey of frequencies of drugresistant tuberculosis simultaneously in 74 countries around the world. Among new TB cases, the median prevalences of overall resistance, monoresistance and multiresistance were $10.2 \%, 7 \%$ and $1.1 \%$ respectively. The prevalence of any monoresistance to streptomycin (SM) was $6.3 \%$, to INH $5.6 \%$, to RPM $1.4 \%$ and to ethambutol (EMB) $0.8 \%$. Analysis among previously treated cases was possible for 65 different settings, and it was found that the median prevalence of any resistance was $18.4 \%$, with eleven settings reporting resistance over $50 \%$, while the median prevalence of multidrug resistance was $7 \%{ }^{24}$. The prevalence of any monoresistance was $11.4 \%$ for SM, $14.4 \%$ for INH, $8.7 \%$ for RMP and 3.5\% for EMB. The WHO study reported resistance data for 39 more settings than in the previous worldwide survey, but in spite of the great variation in the prevalence of TB resistance, it was possible to identify new hotspots with high prevalence of multidrug resistance among new cases.

Previous treatment for tuberculosis is the predominant determinant for the development of MDR-TB ${ }^{520}$. Other risk factors are poor adherence to treatment, the presence of cavitary lesions on chest X-ray and HIV infection ${ }^{61822}$.

In Brazil, culturing and drug susceptibility testing for Mycobacterium tuberculosis strains is not performed on a routine basis and, except for a few studies, the information available on drug resistance is scarce. Information from the worldwide survey coordinated by WHO produced the estimate that, in this country, the national prevalence of acquired resistance to any drug was less than $20 \%$ and multidrug resistance was less than $10 \%$. Primary resistance to any drug was less then $10 \%$ and primary multidrug resistance was less then $1 \%^{24}$. However, Brazil is a very large country with considerable regional differences regarding cultural and socioeconomic conditions. Demographic factors vary also within each region; therefore, heterogeneity is expected in these rates. This has been demonstrated by recent studies such as one performed in São Paulo, Brazil's largest city, with an overall resistance of $15.5 \%$. Among previously treated cases, the prevalence of resistance was $27 \%$ and multidrug resistance was $16.7 \%$. Among new cases, the prevalence of any resistance was $11 \%$ and multidrug resistance was only $1 \%{ }^{21}$.

Cabo de Santo Agostinho, the area of the present study, is an industrial city in northeastern Brazil with an annual incidence of TB of about 60 per 100,000 inhabitants, a $20 \%$ treatment abandonment rate and no information on the frequency of drug resistance until the present study was conducted. The prospective cohort presented here was designed to characterize the drug resistance profile of Mycobacterium strains isolated from patients who were attended for TB investigation at public outpatient clinics and to investigate the risk factors for the development of MDR-TB in the city of Cabo de Santo Agostinho.

\section{MATERIAL AND METHODS}

Study design and population. A cohort study was designed to analyze newly diagnosed TB cases among 773 patients with TB-like respiratory symptoms who attended public outpatient facilities in Cabo de Santo Agostinho between February 2000 and December 2003. At the beginning of this study, the diagnosing of TB in Cabo was centralized. Thus, all suspect TB patients were referred to the Herbert de Souza Health Center, where they were seen by an experienced physician and were invited to have an appointment with the research team. Patients who were seen by the research team and who agreed to participate in this study signed an informed consent statement prior to answering a clinical and epidemiological questionnaire. Sputa were also collected and sent to the Central Laboratory of Pernambuco, which is the reference laboratory of the State of Pernambuco for tuberculosis diagnosis, for bacilloscopy, culturing and resistance testing. Blood samples were sent to the Public Laboratory of Olinda for HIV infection testing.

TB cases were defined by the physician who attended the patient, based on the clinic findings, X-ray and bacilloscopy. Because not all suspected TB cases were examined by the research team, the study population represented a fraction of the TB cases notified during the study period. Unfortunately, it was not possible to make a comparison between notified TB cases that were included in the research database and the overall number of TB cases, because the variables used in the official notification form were different from those in the questionnaire for the present study, other than age and gender, which were similar. Therefore, the results presented here might be not representative of the whole population of TB cases diagnosed in the City of Cabo de Santo Agostinho during the study period.

The Ethics Committee of the Aggeu Magalhães Research Center of the Oswaldo Cruz Foundation approved this study.

Microbiological analysis. Acid-fast bacilli were detected by means of Ziehl-Neelsen staining. The sputa were then decontaminated in accordance with the Petroff method and aliquots of $0.1 \mathrm{ml}$ were placed in Löwenstein-Jensen medium with or without the following antibiotics (Sigma, USA): isoniazid $(0.2 \mu \mathrm{g} / \mathrm{ml})$, streptomycin $(4 \mu \mathrm{g} / \mathrm{ml})$, pyrazinamide $(2 \mu \mathrm{g} / \mathrm{ml})$, ethambutol $(2 \mu \mathrm{g} / \mathrm{ml})$, ethionamide $(2 \mu \mathrm{g} / \mathrm{ml})$, rifampicin $(40 \mu \mathrm{g} / \mathrm{ml})$ or $\mathrm{p}$-nitrobenzoic acid $(500 \mu \mathrm{g} / \mathrm{ml})$. The resistance was determined by the proportion method ${ }^{11}$. In order to increase the bacillary load, all smear-negative or weak positive samples $(+)$ were first grown in the medium without antibiotics for two 
weeks and were then subjected to resistance testing. Fast-growing Mycobacterium was routinely sent to the "Professor Hélio Fraga" National TB Reference Laboratory (Ministry of Health), in Rio de Janeiro, for species identification when these cases were detected by Regional Laboratories within the Public Laboratory network. When a resistant strain was identified on diagnosis, additional sputum was collected two or three months after the treatment began, for follow-up culturing and resistance testing, which have here been termed control cultures.

Data analysis. A database was constructed containing the clinical, epidemiological and laboratory information obtained from all patients under investigation for TB at the "Hebert de Souza" Public Health Center, using the Epi Info 6.04 software. In order to estimate the number of notified TB cases included in the cohort, we also analyzed official records of tuberculosis cases notified from 2000 to 2003, which were provided by the Health Department of Cabo de Santo Agostinho. Both databases were first reviewed to exclude any inconsistent data. Instances of identical data entries within a 30-day period were taken to be duplicates. Cases already undergoing treatment at the beginning of the study period were excluded.

For the resistance analysis, the frequencies of both monoresistance and multiresistance were determined. To allow comparison with international data, multidrug resistant strains were considered to be those that were resistant to at least INH and RMP, as defined by WHO. To determine the resistance index, files from positive culture on diagnosis were first selected and then any files relating to atypical strains that are normally resistant to multiple drugs and thus not a subject of our study were excluded. Nevertheless, the analysis included other files from patients who developed resistance during the course of their treatment, in the understanding that this represented infection by a new strain based on its resistance profile (Figure 1). The resistance was classified as either primary or acquired and, for primary resistance, it was calculated by dividing the number of patients with resistant strains among new cases by the total number of new cases with culturing and resistance testing. For acquired resistance, it was calculated by dividing the number of patients with resistant strains among previously treated cases by the total number of previously treated cases with culturing and resistance testing. The risk ratios for the development of drug-resistant TB were calculated for biological, clinical and socio-economical factors, including history of previous TB and abandonment of treatment, using logistic regression. The significance of these associations was determined using the chi-squared test and p-values, and Yates correction when indicated.

\section{RESULTS}

Cohort characterization. In the present study, 773 symptomatic respiratory cases were investigated for TB (Figure 1). The TB diagnosis was confirmed for 232 cases interviewed, which represented $50.5 \%$ of the 459 notified cases during the study period. The official incidence of TB was around 60 new cases per 100,000 inhabitants per year in the city of Cabo de

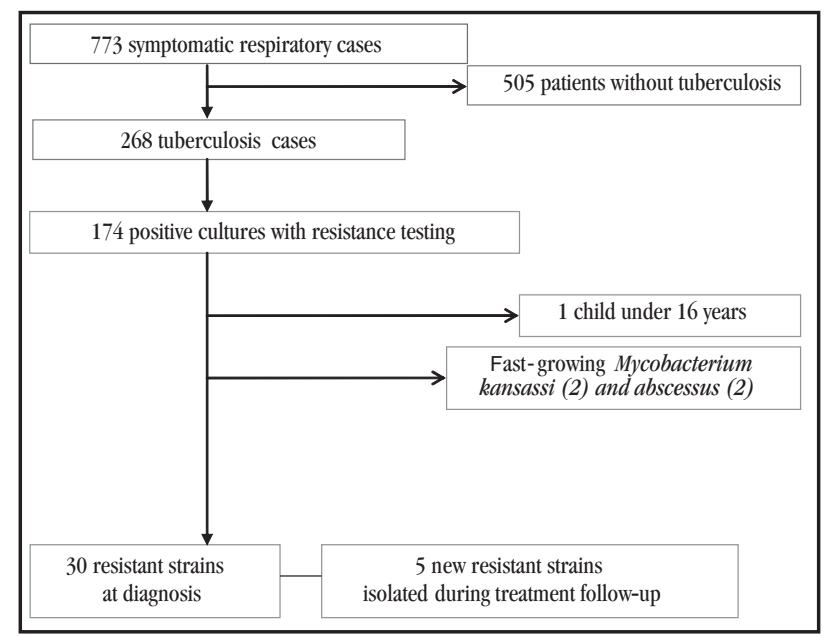

Figure 1 - Selection of the study population. Out of the 268 tuberculosis cases screened, 232 were notified by the Health Department of Cabo de Santo Agostinho during the study period.

Santo Agostinho. Although some fluctuation in the yearly incidence rates could be noticed, no clear upward or downward trend could be observed. A different scenario was apparent when observing two epidemiological characteristics that are associated with drug resistance: abandonment of treatment and disease recurrence (Table 1). A considerably higher number of such events were observed during the first two years of the study, in comparison with the next two years. The mean frequency of abandonment of treatment in 2000-2001 was $15.5 \%$, whereas it decreased to $5 \%$ in 2002-2003 ( $\mathrm{p}=0.004)$. The mean frequencies of TB recurrence were respectively $5.5 \%$ and $2.5 \%$ for these consecutive two-year periods, but this difference was not significant $(\mathrm{p}=0.346)$. It is important to point out that two interventions were implemented by the city's Health Department over the study period: communitybased surveillance in 2002 and decentralization of the healthcare system in 2003. These may have had an influence on the fall in the abandonment rate.

Tuberculosis was more frequent among men than among women (2:1), and the majority of the cases (86.6\%) occurred within the economically active age group (20 to 64 years). Alcohol consumption and smoking were reported by $65.7 \%$ and $59.7 \%$ of the cases respectively. Histories of previous TB contact and anti-TB vaccination were present in $32.5 \%$ and $59.7 \%$ of cases

Table 1 - Coefficients of tuberculosis in Cabo de Santo Agostinbo, 2000-2003.

\begin{tabular}{lrrrrr}
\hline & 2000 & 2001 & 2002 & 2003 & Total \\
\hline Coefficients (per 100,000) & & & & & \\
incidence & 61.4 & 58.8 & 52.3 & 67.3 & \\
prevalence & 81.7 & 76.5 & 61.4 & 80.4 & \\
Total - notified cases & 125 & 117 & 94 & 123 & 459 \\
$\quad$ new cases & 94 & 90 & 80 & 103 & 367 \\
$\quad$ retreatment & & & & & \\
$\quad$ abandonment & 19 & 19 & 3 & 8 & 45 \\
$\quad$ recurrence & 8 & 6 & 3 & 3 & 20 \\
$\quad$ transferred & & & 8 & 9 & 17 \\
$\quad$ not classified & 4 & 2 & & & 6 \\
Total - studied cases & 60 & 70 & 42 & 60 & 232 \\
\hline
\end{tabular}


respectively. During the analysis, information bias was identified when assessing socioeconomic factors using the variables of employment and income, as measured by the minimum official salary in Brazil. There were records of employment activities with no regular incomes, thereby leading to imprecise quantification. About $70 \%$ of the patients were literate.

Culturing results and bacteriological characterization of mycobacterial isolates. Among all of the 773 suspected cases, Mycobacterium was isolated by means of Löwenstein-Jensen culturing in 181 (23.4\%) samples, while 559 (72.3\%) were negative cultures, 10 (1.3\%) presented technical problems during the isolation procedure and $23(3 \%)$ were not subjected to culturing. Among the 181 positive cultures, $137(75.7 \%)$ were positive to alcohol-acid staining while 44 (24.3\%) were smear-negative. All the negative cultures were also smear-negative. The bacilloscopy performed by the State Laboratory had sensitivity of $75.7 \%$, specificity of $100 \%$, positive predictive value of $100 \%$ and negative predictive value of $92.7 \%$.

Thirty out of the 169 isolate samples on which resistance testing was performed at diagnosis showed resistance to at least one drug. However a further five isolates were obtained from patients undergoing treatment and were included in the resistance analysis because they were judged to represent new strains (their resistance testing showed profiles that differed from what was seen at the diagnosis). However, it was not possible to distinguish whether this was due to new infection or to selection of a mutant strain during the course of the treatment. All 35 Mycobacterium tuberculosis isolates were resistant to at least isoniazid. Resistance to at least rifampicin was found in 24 (14\%) of the isolates. Monoresistance to isoniazid occurred in seven (4\%) of the isolates. No monoresistance to rifampicin was found, and an association between isoniazid and rifampicin occurred in 16 (9\%) of the cases. Resistance to isoniazid and rifampicin, or multidrug resistance, occurred in 24 (14\%) of the cases. The frequency of primary resistance was $14 \%$ and of acquired resistance was 50\%. The frequency of MDR-TB among new cases was $8 \%$ and among previously treated cases was $40 \%$. The proportions of resistance among previously treated cases and new cases were 3.6 for monoresistance, 2.6 for two-drug resistance and 8.0 for resistance to three or more drugs (Table 2). Secondary and primary resistance to the specific combination of isoniazid and rifampicin was $20 \%$ and $6.3 \%$ with a ratio of 3.2. In a recent visit to the Cabo Health Center, we investigated the outcomes from the patients with drug-resistant $\mathrm{TB}$ reported here and found that cases of monoresistance to isoniazid either presented recurrence or were cured using scheme 1. We also observed that most multidrug-resistant cases completed the six-month treatment of scheme 1 , even though the culture results were provided by the research team (data not shown).

Risk factors for developing drug-resistant tuberculosis. Comparison of the biological, clinical and epidemiological characteristics of the patient groups with drug-sensitive and resistant isolates showed that there were no statistically significant differences between the groups regarding frequencies of age, gender, literacy, being employed/unemployed, income category or presence of clinical symptoms. However, previous TB treatment and/or presenting a history of abandoning treatment were strongly associated with resistance (Table 3). The risk of developing MDRTB was higher for those who reported previous TB treatment
Table 2- Frequency of drug-resistant Mycobacterium tuberculosis among cases diagnosed in Cabo de Santo Agostinbo, 2000-2003.

$$
\begin{array}{ccccc}
\text { Number } & \text { Frequency } & \text { Prevalence } & \text { Prevalence } & \text { Ratio of } \\
& (\%) & \text { of primary } & \text { of acquired } & \text { acquired } \\
& & \text { resistance } & \text { resistance } & \text { to primary }
\end{array}
$$

\begin{tabular}{lrrrrr} 
& \multicolumn{1}{c}{} & & resistance \\
\hline $\begin{array}{l}\text { Mycobacterium } \\
\text { tuberculosis isolates }\end{array}$ & 174 & & 13.9 & 50.0 & 3.6 \\
\hline Any resistance & 35 & 20 & & & - \\
isoniazid (H) & 35 & 20 & - & - & - \\
streptomycin (S) & 7 & 4 & - & - & - \\
rifampicin (R) & 24 & 14 & - & - & - \\
ethambutol (E) & 5 & 3 & - & - & - \\
ethionamide (Et) & 4 & 2 & - & - & - \\
pyrazinamide (Z) & 1 & 1 & - & - & - \\
H, R (MDR) & 24 & 14 & 8.3 & 40.0 & 4.8 \\
H, S & 6 & 3 & - & - & - \\
\hline Specific resistance & & & & & 3.6 \\
\hline H & 7 & 4 & 2.8 & 10.0 & 2.6 \\
H, S & 2 & 1 & 9.0 & 23.3 & \\
H, E & 1 & 1 & & & \\
H, Et & 1 & 1 & & & \\
H, R & 16 & 9 & & & \\
H, R, S & 3 & 2 & 2.1 & 16.7 & \\
H, R, E & 1 & 1 & & & \\
H, R, E, Et & 2 & 1 & & & \\
H, R, S, E & 1 & 1 & & & \\
H, R, Z, S, Et & 1 & 1 & & & \\
\hline
\end{tabular}

\begin{tabular}{|c|c|c|c|c|c|c|}
\hline & \multicolumn{4}{|c|}{ Tuberculosis* } & \multirow[b]{3}{*}{ Risk relative } & \multirow[b]{3}{*}{$\mathrm{p}$} \\
\hline & \multicolumn{2}{|c|}{ resistant } & \multicolumn{2}{|c|}{ not resistant } & & \\
\hline & $\mathrm{n}^{\underline{0}}$ & $\%$ & $\overline{\mathrm{n}^{0}}$ & $\%$ & & \\
\hline \multicolumn{7}{|l|}{ Gender $\leq 34$} \\
\hline yes & 20 & 21.3 & 74 & 78.7 & $1.13[0.62-2.07]$ & 0.82227 \\
\hline no & 15 & 18.8 & 65 & 81.3 & & \\
\hline \multicolumn{7}{|l|}{ Sex female } \\
\hline yes & 11 & 20.8 & 42 & 79.2 & $1.05[0.55-1.98]$ & 0.94727 \\
\hline no & 24 & 19.8 & 97 & 80.2 & & \\
\hline \multicolumn{7}{|c|}{ Alcohol consumption } \\
\hline yes & 22 & 18.6 & 96 & 81.4 & $0.76[0.42-1.39]$ & 0.49836 \\
\hline no & 13 & 24.5 & 40 & 75.5 & & \\
\hline \multicolumn{7}{|l|}{ Employment } \\
\hline yes & 12 & 19.7 & 49 & 80.3 & $1.05[0.53-2.11]$ & 0.94345 \\
\hline no & 14 & 18.7 & 61 & 81.3 & & \\
\hline \multicolumn{7}{|l|}{ Income $\leq 2$} \\
\hline \multicolumn{7}{|c|}{ national minimum } \\
\hline \multicolumn{7}{|l|}{ salaries } \\
\hline yes & 25 & 22.7 & 85 & 77.3 & $1.70[0.82-3.54]$ & 0.20160 \\
\hline no & 8 & 13.3 & 52 & 86.7 & & \\
\hline \multicolumn{7}{|l|}{ Illiterate } \\
\hline yes & 11 & 27.5 & 29 & 72.5 & $1.51[0.81-2.81]$ & 0.28995 \\
\hline no & 8 & 18.2 & 108 & 81.8 & & \\
\hline \multicolumn{7}{|c|}{ Previous treatment } \\
\hline yes & 15 & 50 & & 50 & $3.60[2.09-6.19]$ & 0.00002 \\
\hline no & 20 & 13.9 & 15 & 50.0 & & \\
\hline Abandonment & & & 86 & & & \\
\hline yes & 10 & 52.6 & 9 & 47.4 & $3.26[1.87-5.70]$ & 0.00057 \\
\hline no & 25 & 16.1 & 130 & 83.9 & & \\
\hline
\end{tabular}

Table 3-Risk factors for the development of drug-resistant tuberculosis in Cabo de Santo Agostinbo.

*missing data was subtracted before statistical analysis.

data relating to retired status was excluded from employment variable. 
$(\mathrm{RR}=4.9 ; 95 \% \mathrm{CI}=2.48-9.77 ; \mathrm{p}=0.00004)$ and treatment abandonment ( $\mathrm{RR}=3.7 ; 95 \% \mathrm{CI}=1.82-7.56 ; \mathrm{p}=0.0033)$. Among the 232 notified TB cases, 185 underwent HIV testing: three samples were found to be positive and 13 were inconclusive, giving a frequency of TB-HIV coinfection of $1.6 \%$. None of the MDR-TB cases were positive for HIV infection.

\section{DISCUSSION}

In the present study, MDR-TB, defined as resistance to at least isoniazid and rifampicin was responsible for $14 \%$ of the overall number of TB cases and 69\% of the drug-resistant TB cases in the City of Cabo de Santo Agostinho. The prevalence of MDR-TB was as high as $8 \%$ among new TB cases and $40 \%$ among patients with previous treatment for the disease.

There is still not enough data published on MDR-TB in Brazil. A retrospective study covering one decade (1990-1999) in Ceará, another city in northeastern Brazil, reported an acquired MDR-TB frequency of $0.6 \%^{2}$. In Rio de Janeiro, a Brazilian city with high demographic density and a poor situation regarding tuberculosis, the frequency of acquired MDR-TB in the year 1994 was 5.2\% ${ }^{13}$. More recently, a study in a district of São Paulo, Brazil's largest city, reported a frequency of $16.7 \%$ for the year $2000-2002^{21}$. Such differences in MDR-TB prevalence as observed in Brazil have also been reported in other countries. One example is Bangladesh, which has an overall MDR rate of 5.5\% but considerably higher levels in the regions of Dhara (14.3\%) and Matlab $(27.3 \%)^{25}$. The resistance rate observed in Cabo is, on the other hand, similar to rates reported in some regions where drug resistance and MDR have been reported to be a major concern such as $54 \%$ in Lithuania $^{4}, 40.2 \%$ in Uzbekistan ${ }^{3}$ and 30.5\% in Taiwan ${ }^{7}$.

The primary MDR level of $8 \%$ was also higher than the $0.1 \%$ observed during the national survey ${ }^{23}$ and was comparable with the reported 5.8\% in Mozambique ${ }^{14}, 6 \%$ in Colombia ${ }^{12}, 9.3 \%$ in Lithuania ${ }^{4}$ and $13 \%$ in Uzbekistan ${ }^{3}$. It has been reported that countries with cure rates greater than $85 \%$ have primary MDRTB levels of less than 5\% and that the prevalence of MDR-TB is ten times higher among previously treated patients than in new cases $^{16}$. This reinforces the idea that primary MDR are related to failing TB control programs, while secondary MDR seems to be a consequence of lack of treatment compliance. In Cabo, the cure rate is currently still below $85 \%$. Before the intervention of the TB Control Program in 2002, the rate of abandonment of treatment was as high as $15 \%$. These epidemiological characteristics may have contributed towards the high levels of both types of resistance in the region, thereby leading to the present prevalence of primary MDR-TB of $8 \%$.

One important finding was the ratio between acquired and primary resistance when considering resistance to one or more drugs: this ratio was 3.6 for monoresistance, 2.6 when there was resistant against any two drugs and 8.0 against three or more drugs. The greater this ratio was, the higher the acquired resistance was, which could be explained by the historical high frequency of treatment abandonment in Cabo, even though the incidence and prevalence rates were shown to be relatively stable (Table 1).
A retrospective study on the prevalence of resistance to at least one drug was performed in Turkey, covering three decades starting in 1972. In this, an increase of $1.4 \%$ in the ration between acquired and primary MDR was observed for each decade. This was accompanied by decreases in the ratio between acquired and primary resistance to any drug over the three decades (6.5, 5.1 and 4.6), thus suggesting that the increase in MDR prevalence was due to rises in primary resistance . $^{8}$

Although the time frame of our study was too short for such an analysis, a lower ratio of acquired and primary MDR-TB (4.8) was observed in comparison with the ratio for resistance to three or more drugs (8.0). In the present study, the ratio between acquired and primary combined resistance to INH and RPM was $3.2,4.8$ or 8.0 , depending on whether the cases were only resistant to INH and RMP, were resistant to at least INH and RMP or were associated with resistance to other drugs. This observation could be interpreted to mean that specific resistance to INH and RMP might be occurring as a consequence of lack of effective TB-control strategy in Cabo. This would lead to an increase in the primary resistance to specific drug combinations, thereby lowering the ratio in relation to that of MDR-TB.

We found a level of primary monoresistance against INH of $14 \%$ and absence of monoresistance to RMP. The observed pattern of resistance differed from the data presented in a recent review of 21 studies on the levels of primary resistance among Mycobacterium tuberculosis isolates in India, a country that accounts for $30 \%$ of TB cases worldwide. A high median prevalence of primary resistance to INH of $16.5 \%(3.2-32.9 \%)$ was observed, in comparison with the level of $2.85 \%$ (0-11.8\%) for RMP and 1.5\% (0-5.3\%) for combined INH and RMP resistance ${ }^{17}$. A population study in Rio de Janeiro, in 1994 showed a similar behavior of resistance frequency: $4.6 \%$ and $0.9 \%$ for primary monoresistance to INH and RMP respectively and $0.7 \%$ for the combined resistance ${ }^{13}$. This was because the mutation rate for genes involved in INH resistance was higher than that reported for rifampicin resistance. Double mutation is therefore a rare event: the development of combined resistance to INH and RMP is unlikely to occur if the patient receives adequate care.

Cases of acquired resistance are reported to be due to inappropriate or irregular drug supply or to patient behavior manifested by nonadherence to treatment, thereby favoring the selection of spontaneously mutated Mycobacterium tuberculosis strains ${ }^{19}$. In the present study, the patients who had a history of previous treatment at the time of diagnosis had a five times greater risk of presenting MDR-TB. This association has also been found by other authors ${ }^{5620}$. A history of abandonment of treatment was also found to lead to four times greater risk of having MDR-TB in Cabo. In addition to the frequency of abandonment of treatment in Cabo of 15\% when the study began, we found a high frequency of alcohol consumption, which has been reported to be a risk factor for treatment abandonment ${ }^{1}$.

The frequency of HIV coinfection in TB patients in Cabo was 1.6\%. In Brazil, the rate of TB coinfection in patients with HIV/AIDS has been estimated to be $3-4 \%$ of cases ${ }^{9}$. All the patients with HIV infection reported here had TB caused by drug-susceptible 
Mycobacterium. Development of drug-resistant tuberculosis was, however, associated with HIV in five of the seven European countries reporting this variable in a recent published systematic reviews.

In conclusion, primary and secondary resistance to both INH and RMP was high in Cabo, thus suggesting that MDR strains are in circulation. This demonstrates the patchy nature of the degree of MDR and suggests that it may be very important to study regional variations. Culturing and resistance testing is recommended for all previously treated cases of TB, for cases without clinical improvement after two months of therapy and for new patients with close contacts that present MDR-TB. Therefore, the main challenge for TB control programs is to provide good laboratory support and to ensure that physicians are informed about the results from the laboratory tests. Decisions regarding adjustment of TB treatment schedules should be discussed in all cases with monoresistance to INH, including the addition of new drugs to the therapeutic schedule and using longer treatment periods. Application of molecular methods to genotype strains, with identification of mutations causing drug resistance among patients who do not respond well to treatment, could be an additional tool for avoiding delayed cure, transmission of MDR strains and mortality.

\section{ACKNOWLEDGEMENTS}

We thank the Health Department of Cabo de Santo Agostinho for access to the patients and the Central Laboratory of Pernambuco for their help in performing the microbiological work. We are also grateful to Clenildo Filho, Fábio de Souza, Márcio Bezerra and Leonardo Dutra for their technical help.

\section{REFERENCES}

1. Albuquerque MFPM, Ximenes RAA, Lucena-Silva N, Souza WV, Dantas AT, Dantas OMS, Rodrigues LC. Factors associated with treatment failure, dropout, and death in a cohort of tuberculosis patients in Recife, Pernambuco State, Brazil. Caderno de Saúde Pública 23: 105-114, 2007.

2. Barroso EC, Rodrigues JLN, Pinheiro VGF, Campelo CL. Prevalência da tuberculose multirresistente no Estado do Ceará, 1990-1999. Jornal de Pneumologia 27: 310-314, 2001.

3. Cox HS, Orozco JD, Male R, Ruesch-Gerdes S, Falzon D, Small I, Doshetov D, Kebede Y, Aziz M. Multidrug-resistant tuberculosis in Central Asia. Emerging Infectious Diseases 10: 865-872, 2005.

4. Dewan P, Sosnovskaja A, Thomsen V, Cicenaite J, Laserson K, Johansen I, Davidaviciene E, Wells C. High prevalence of drug-resistant tuberculosis, Republic of Lithuania, 2002. The International Journal of Tuberculosis and Lung Disease 9:170-174, 2005.

5. Faustini A, Hall AJ, Perucci CA. Risk factors for multi-drug resistant tuberculosis in Europe: a systematic review. Thorax 61:158-163, 2006.

6. Granich RM, Oh P, Lewis B, Porco TC, Flood J. Multi-drug resistance among persons with tuberculosis in California, 1994-2003. Journal of American Medical Association 293: 2732-2739, 2005.
7. Hsueh PR, Liu YC, So J, Liu CY, Yang PC, Luh KT. Mycobacterium tuberculosis in Taiwan. Journal of Infection 52:77-85, 2006.

8. Kart L, Altin R, Tor M, Gulmez I, Oymak SF, Atmaca HM, Erdem F. Anti-tuberculosis drug resistance patterns in two regions of turkey: a retrospective analysis. Annals of Clinical Microbiology and Antimicrobials 16: 1-6, 2002.

9. Kritski AL, Ruffino-Netto A. Health sector reform in Brazil: impact on tuberculosis control. The International Journal of Tuberculosis and Lung Disease 4: 622-626, 2000 .

10. Min SM, Kelly P, Byrne C, Clancy L. Antibiotic resistant tuberculosis and bovine tuberculosis in an Irish hospital population (1991 to 2001). Irish Medical Journal 98: 38-40, 2005.

11. Ministério da Saúde do Brasil. Tuberculose-guia de vigilância epidemiológica/ elaborado pelo Comitê Técnico-científico de Assessoramento à Tuberculose e Comitê Assessor para Co-infecção HIV-Tuberculose. Ministério da Saúde/ Fundação Nacional da Saúde, Brasília, 2002.

12. Moreira CA, Hernandez HL, Arias NL, Castano MC, Ferro BE, Jaramillos E. Initial drug resistance as a threat for tuberculosis control: the case of Buenaventura, Colombia. Biomedica 1: 73-79, 2004.

13. Natal S, Valente JG, Sánchez AR, Penna MLF. Resistência a isoniazida e rifampicina e história de tratamento anterior para tuberculose. Caderno de Saúde Pública 19: 1277-1281, 2003

14. Nunes EA, De Capitani EM, Coelho E, Joaquim OA, Figueiredo IRO, Cossa AM, Panunto AC, Carvalho-Ramos M. Patterns of anti-tuberculosis drug resistance among HIV-infected patients in Maputo, Mozambique, 2002-2003. The International Journal of Tuberculosis and Lung Disease 9: 494-500, 2005.

15. Ormerod LP. Multi-drug resistant tuberculosis (MDR-TB): epidemiology, prevention and treatment. British Medical Bulletin 73-74: 17-24, 2005.

16. Pablo-Méndez A, Gowda DK, Frieden TR. Controlling multidrug-resistant tuberculosis and access to expensive drugs: a rational framework. Bulletin of the World Health Organization 80: 489-500, 2002.

17. Paramasivan $\mathrm{CN}$, Venkataraman P. Drug resistance in tuberculosis in India. Indian Journal of Medical Research 120: 377-386, 2004.

18. Pozniak A. Multidrug-resistant tuberculosis and HIV infection. Annals New York Academy of Science 953: 192-198, 2001.

19. Raviglione MC, Gupta R, Dye CM, Espinal MA. The burden of drug-resistant tuberculosis and mechanisms for its control. Annals New York Academy of Science 953: 88-97, 2001.

20. Surucuoglu S, Ozkutuk N, Celik P Gazi H, Dinc G, Kurutepe S, Koroglu G, Havlucu Y, Tuncay G. Drug-resistant pulmonary tuberculosis in western Turkey: prevalence, clinical characteristics and treatment outcome. Annals Saudi Medicine 25: 313-318, 2005.

21. Telles MA, Ferrazoli L, Waldman EA, Giampaglia CM, Martins MC, Ueki SY, Chimara E, Silva CA, Cruz V, Waldman CC, Heyn I, Hirono IU, Riley LW. A population-based study of drug resistance and transmission of tuberculosis in an urban community. The International Journal of Tuberculosis and Lung Disease 9: 970-976, 2005.

22. Vanacore P, Koehler B, Zacchini F, Bassetti D, Antonucci G, Hipólito G, Girardi E, GISTA-SIMIT Study Group. Drug-resistant tuberculosis in HIV-infected persons: Italy 1999-2000. Infection 32: 328-332, 2004.

23. World Health Organization. Anti-TB drug resistance in the world. The WHO/ IUATLD global project on anti-tuberculosis drug resistance surveillance. WHO, Geneva, 1997.

24. World Health Organization. Anti-TB drug resistance in the world. Prevalence of drug resistance. The WHO/IUATLD global project on anti-tuberculosis drug resistance surveillance. WHO, Geneva, 2004.

25. Zaman K, Rahim Z, Yunus M, Arifeen SE, Baqui AH, Sack DA, Hossain S, Banu S, Islam MA, Ahmed J, Breiman RF, Black RE. Drug resistance of Mycobacterium tuberculosis in selected urban and rural areas in Bangladesh. Scandinavian Journal of Infectious Diseases 37: 21-26, 2005. 